\title{
Synthesis and Characterization of Poly(styrene)- block-Poly(acrylic acid) and Organoclay Based Hybrid Composite Materials
}

\author{
Sajid Haleem Soomro \\ Institute of Chemistry, \\ Shah Abdul Latif University, \\ Khairpur, Pakistan \\ sajidsoomro80@gmail.com
}

\author{
Muhammad Sanaullah \\ Institute of Geology, \\ University of the Punjab, \\ Lahore, Pakistan \\ sana.ullah.geo@pu.edu.pk
}

\author{
Noor Zaman \\ National Center of Excellence in Analytical \\ Chemistry, University of Sindh, \\ Jamshoro, Pakistan \\ noorzamanshar@yahoo.com
}

\author{
A. Q. Laghari \\ Dpt. of Chemical Engineering, \\ Mehran University of \\ Engineering \& Technology, \\ Jamshoro, Pakistan \\ engrchemi@gmail.com
}

\author{
Nabi Bux Jalbani \\ Dpt. of Chemical Engineering, \\ Quaid-e-Awam University of \\ Engineering, Science and \\ Technology, Pakistan \\ n.bux@quest.edu.pk
}

\author{
Kamran Mirza \\ Institute of Geology, \\ University of the Punjab \\ Lahore, Pakistan \\ kamran.geo@pu.edu.pk
}

\author{
Ejaz Ahmed Abbasi \\ Institute of Geology, University \\ of Azad Jammu and Kashmir, \\ Muzaffarabad, AJK, Pakistan \\ ejazahmed990@gmail.com
}

\begin{abstract}
Polystyrene (PS) and polyacrylic acid (PAA) are organic and synthetic polymers. Generally, a composite needs a matrix and reinforcement. Montmorillonite (MMT) is used as reinforcement to form a hybrid matrix by ultra-sonication. Block polymers of PS and PAA were first synthesized and then hybrid nanocomposite material of synthesized block polymeric matrix was fabricated by incorporating organoclay as reinforcement through 6 and 12 hours of ultra-sonication. The characterizations of the hybrid nanocomposite material were done by UV-visible and infrared (IR) spectroscopy, which confirm the successful synthesis of PS and PAA block polymer. The UV-visible spectroscopic measurements revealed that hybrid composite material is different from PS-block-PAA and that absorbance increases on increasing concentration. After successful ultrasonication the hybrid composite is analyzed through scan electron microscopy (SEM) regarding surface morphology, distribution, uniformity, porosity, matrix compatibility, and matrix reinforcements. Sonication (for 6 hours) demonstrates a heterogeneous porous surface and an increase in roughness and porosity of surface morphology at different optical zooms.
\end{abstract}

Keywords-poly(acrylic acid); poly(styrene); block polymer; montmorillonite (MMT); exfoliation

\section{INTRODUCTION}

A polymeric composite material is made out of two distinct parts, network and filler. It is also called fortification or dispersed stage $[1,2]$. It might come in the form of strands (fibers), sheets or particles. However, sometimes extra mixtures are utilized. The properties of composites depend on size, shape, orientation, relative amount, distribution of particles, constituent phase, and geometry of dispersed phase [3]. The dispersed medium is liable for further structure improvement.
In order to improve the interaction between filler and matrix, compatibilizers can be added which have expressive effect on material cohesion and properties. Due to their unique advantages composite materials are used in various technical applications [4]. The combination of synthetic or natural inorganic filler makes up polymer matrix composites (PMCs). PMCs comprise resins as matrix. Metal matrix composites (MMCs) can enhance properties like tensile strength, yield stress and high impact resistance. MMCs usually are alloys of $\mathrm{Al}$ and $\mathrm{Mg}$ using silicon carbide fibers [5]. Polymers have been with us and other species from the beginning, they formulate the basic building blocks of life [6]. Polymers like polyethylene, PS, plastics and nylon are obtained by synthetic methods. The polymeric products are widely abundant in nature [7, 8]. Nano scale structuring material has unique properties. The physical and chemical properties of nano scale materials significantly differ from the ones at large scale. Filler alternative additive nanoparticles utilized within polymers for different impacts are an expanded investment [9]. Regarding physical structure, nanofillers might be characterized into their substance nature, yet they are arranged in view of the particle states. Constituents have at least one dimension in the nanometer scale [10]. The collaboration amongst nanoclays and polymers can deliver two sorts of nanocomposites. The primary type possess intercalated nanocomposites, which are framed because of the infiltration of polymer chains between the layers of the clay, shaping a multilayer structure with rotating layers of polymer and clay [11].

\section{MATERIALS AND METHODS}

It is logical to investigate spectrum and quantum properties regarding ionized electronic conduction of fabricated high sub- 
atomic weight novel composite and nanocomposite materials. It is fundamental to acquire and investigate optical and quantum analysis with expanded ultraviolet and close infrared spectroscopy. The hybrid composite material of Polystyreneblock-Polyacrylic acid (PS-b-PAA) is characterized by FT-IR, SEM and UV-Visible spectrophotometer. Analyses are done in order to know the shifting of functional groups and main bond vibrations and to accumulate and confirm the nano-fabrication.

\section{A. UV-Visible Spectroscopy}

UV-visible spectroscopy is a valuable apparatus to scan the natural atoms that incorporate in saturated and unsaturated compounds like aromatic compound, alkanes, alkenes, alkynes, ketones and aldehydes for various distinct wavelengths. It delivers change from pre-synthesis to post-synthesis [13].

\section{B. FT-IR Spectroscopy}

Polymers and composite materials would be also examined by FT-IR spectroscopy. The limitation and utilitarian of instrument is in terms of vibrational frequencies of single wavelength to various wavelengths of polymers and composite materials. Bonding and circulation of polymerized and copolymerized monomeric units in chains is done with the aid of hydrogen bonding [15]. All the inner and outer composition can be revealed by the FT-IR technique. The created crosslinked and polymer and nano-composite blocks are identified in terms of wave numbers and bands of vibrations.

\section{Scanning Electron Microscopy (SEM)}

SEM is a helpful system to examine the surface morphology of a composite material. SEM runs through a PC program, the pictures of SEM are seen on CRTs arranged at electronic reassure in addition to EFI pictures [16].

\section{Ultra Sonication}

Ultra sonication is a process which converts electrical signals into physical vibrations. Ultra sonication is mostly done by sonicator devices which work on a mechanism in which sound waves are converted into mechanical waves. Sonication has various impacts on physical and chemical properties. Sonication can be utilized to speed disintegration, by breaking intermolecular associations. It is particularly helpful when it isn't attainable to mix the given substance, as with NMR tubes [12].

\section{E. Centrifuge}

A centrifuge is deployed for the detachment of particles of matter whether it is solid, liquid or gas. A centrifuge works on the application of centrifugal force for the sedimentations of heterogeneous mixture. It is a device for isolating particles from a solution as indicated by their size, shape, thickness, density, medium consistency, and rotor speed. In a mixture, particles whose thickness is higher than that of the dissolvable sink and particles that are lighter than it glide to the top. The more prominent the distinction in density, the speedier they move. If there is no distinction in thickness (isopycnic conditions), the particles remain steady [14].

\section{F. Chemicals and Reagents}

Following chemicals were utilized without sterilization. Refined water was utilized for arrangement of tests.
- Polystyrene $99 \%$ pure

- Poly acrylic acid $99 \%$ pure

- MMT nanoclay

- Tetra hydrofuran (THF) (solvent) $98 \%$

\section{G. Synthesis of PS-b-PAA \MMT Clay Composite}

For the synthesis of PS-b-PAA and MMT clay based hybrid composite materials, $3 \mathrm{~g}$ of PS were dissolved in $30 \mathrm{ml}$ of THF and were shaken till the viscous nature of solution increased. Ten $\mathrm{ml}$ from the resulting solution were mixed with $10 \mathrm{ml}$ of PAA and the mixture was sonicate for 30 minutes to form the block polymer of PS-PAA. The appearance of light brownish color justifies the reaction proceedings. After careful monitoring we added $1 \mathrm{~g}$ of MMT clay in the brownish colored solution under continuous stirring for 1 hour. The solution was sonicated for $6 \mathrm{~h}$ to $12 \mathrm{~h}$. In spite of the fact that the specimens were gathered for different hours (3, 6, 9 and 12 hours), to get an even checked trial contrasts and for successful incorporation 6 hours and 12 hours ultra-sonicated arrangements were sufficient to indicate clear nanofabrication. After ultrasonication the color consistently changes to white colored solid as well as white viscous solution formed that came about because of development of composite via reinforcement and matrix. The nanocomposite hybrid material changes its properties and behavior with the designed structure. It was additionally affirmed with the assistance of FT-IR and SEM results.

\section{RESULTS AND DISCUSSION}

\section{A. Spectrophotomteric Study \& UV-Visible Measurement}

The qualitative studies of PS-PAA and their hybrid composite material by incorporation of MMT organoclay, were studied by UV-Visible spectroscopy which gives strong indication for the successful incorporation of organoclay in the matrix. The pure block copolymer of PS-PAA was scaned in $300 \mathrm{~nm}-700 \mathrm{~nm}$ and its maximum absorbace was found at $479 \mathrm{~nm}$. After, the different solutions of composite material i.e, $10 \%, 20 \%, 30 \%, 40 \%$, and $50 \%$ were scanned at $479 \mathrm{~nm}$ to determine absorbance. It was found that the absorbance increases with the increase of concenration, almost in linear relation, except minute deviation observed due to expected impurities during the preperation of the solutions. The results are depicted in Table I. Results show that absorbance increases with the increase of concentration of hybrid composite material (Figure 1). This indicates the formation of hybrid composite material between block copolymer of PS-PAA and organoclay. Hybrid composite material should differ from its polymer matrix and on increasing concentration the absorbance should increase in linear relationship.

TABLE I. PS-PAA CONCENTRATION AND ABSORBANCE

\begin{tabular}{|c|c|c|}
\hline Concentration \% of HCM & Absorbance & \multirow{2}{*}{$\lambda_{\max }$} \\
\hline $10 \%$ & 0.769 & \multirow{3}{*}{$479 \mathrm{~nm}$} \\
\hline $20 \%$ & 0.834 & \\
\hline $30 \%$ & 0.918 & \\
\hline $40 \%$ & 1.091 & \\
\hline $50 \%$ & 1.423 & \\
\cline { 1 - 2 } & 1.178 & \\
\hline Pure block co-polymer PS-PAA & \\
\hline
\end{tabular}




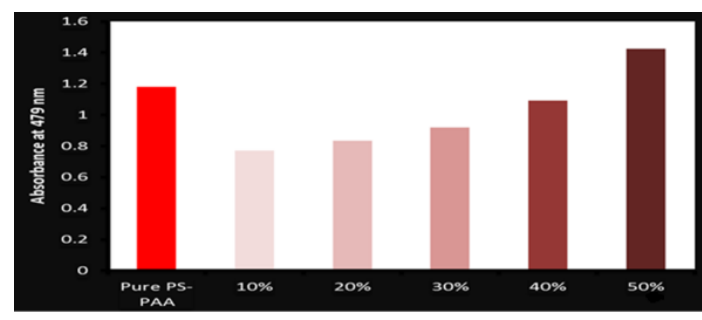

Fig. 1. UV-Visible spectroscopy results

\section{B. FT-IR of PS- $b-P A A$}

FT-IR spectrum of PS-b-PAA shows peak at $2985 \mathrm{~cm}^{-1}$ for $\mathrm{CH}_{3}$ symmetric stretch vibrations (Figure 2). The peak at $1721 \mathrm{~cm}^{-1}$ corresponds to $\mathrm{C}=\mathrm{O}$ stretching, confirming the presence of PAA which was initially present in PAA at $1696 \mathrm{~cm}^{-1}$ and $\mathrm{C}=\mathrm{C}$ aromatic peaks in phenyl ring of polystyrene were initially present at $1601 \mathrm{~cm}^{-1}-1458 \mathrm{~cm}^{-1}$ which was shifted to $1640 \mathrm{~cm}^{-1}-1451 \mathrm{~cm}^{-1}$. This confirms that both characteristic peaks of acrylic and styrene are present in the block of composite material. There also exist $\mathrm{C}-\mathrm{O}$ for carboxylic acid at $1275 \mathrm{~cm}^{-1}-1175 \mathrm{~cm}^{-1}$ and the broadening of peaks at $>3000 \mathrm{~cm}^{-1}$ justifies the presence of $\mathrm{H}$-bonding.

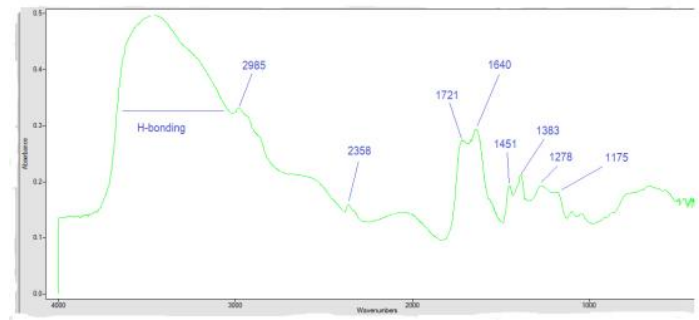

Fig. 2. FT-IR spectrum of PS-b-PAA

\section{FT-IR of 6 Hour Sonicated Hybrid Composite}

FTIR spectrum was recorded after 6 hours sonication of the hybrid composite. The spectrum shows the characteristic peak at $1031 \mathrm{~cm}^{-1}$ for $\mathrm{Si}-\mathrm{O}$ stretching of tetrahedral silicate layers of the surface modified clay. The appearance of this peak shows that polymeric chains are separated due to the incorporation of nano clay. This can also be justified by the significant decrease in the intensity of Si-O. The peaks at $2925 \mathrm{~cm}^{-1}-2850 \mathrm{~cm}^{-1}$ are for $-\mathrm{CH}$ asymmetric and symmetric stretching respectively, while the peak at $1703 \mathrm{~cm}^{-1}$ for $\mathrm{C}=\mathrm{O}$ stretching shows the existence of PAA. This confirms the configuration of nanocomposites (Figure 3).

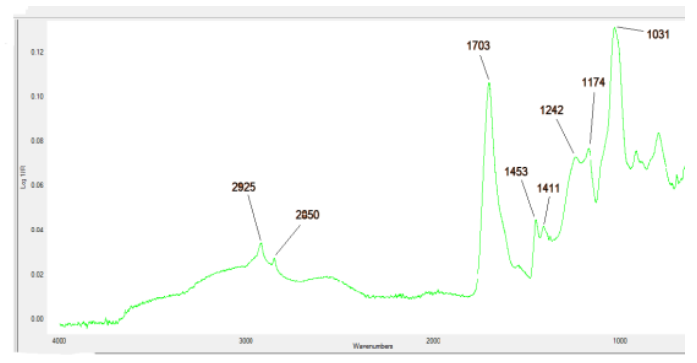

Fig. 3. FT-IR of 6 hour sonicated hybrid composite

\section{FT-IR of 12 Hour Sonicated Hybrid Composite}

After 12 hour sonication of hybrid composite, FTIR shows difference in the peak positions. The peaks at $2915 \mathrm{~cm}^{-1}$ and $2853 \mathrm{~cm}^{-1}$ are for $\mathrm{sp}^{2}$ and $\mathrm{sp}^{3} \mathrm{C}-\mathrm{H}$ stretch and the peak at $1707 \mathrm{~cm}^{-1}$ for $\mathrm{C}=\mathrm{O}$ stretching. The peak at $1015 \mathrm{~cm}^{-1}$ is attributed to Si-O stretching of MMT. The peak of MMT is still visible in the hybrid composite; showing that MMT has been dispersed in the polymeric matrix. As the hybrid composite was prepared through sonication, MMT has not been intercalated in the polymer matrix but rather exfoliated (Figures 4 and 5).

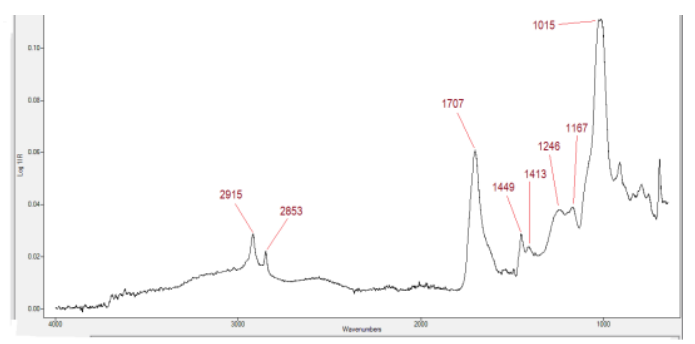

Fig. 4. FT-IR of 12 hour sonicated hybrid composite

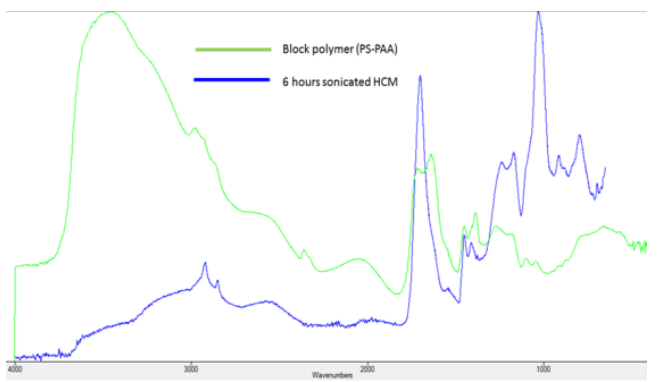

Fig. 5. Comparative FT-IR of block polymer PS-PAA \& 6 hour sonicated hybrid composite material

\section{E. IR Characterization of Composite Material Comparative}

The appearance of peak at $1031 \mathrm{~cm}^{-1}$ is attributed to Si-O bond, which is a characteristic peak for organoclay. The successful incorporation of organoclay can be seen by observing the decrease in H-bonding. The two layers of block are strongly merged with H-bonding. After incorporation of organoclay between two layers of block, an obvious decrease in H-bonding is observed.

\section{F. SEM Analysis of PS-PAA Hybrid Composite (6-Hour Ultra-Sonication)}

SEM study of PS-PAA hybrid composite incorporated with MMT reveals that the morphology of hybrid composite material shows porous surface. Circulation and position of lattices over the surface show the unjumbled spines with somewhat rocky shape (Figure 6). It further reports the nanofabrication with reference to the successful incorporation of organoclay over the composite material with different interfacial faces and edges.

G. SEM Analysis of PS-PAA Hybrid Composite (12-Hour Ultra-Sonication)

SEM study of PS-PAA hybrid composite material 
incorporated with organoclay reveals that the morphology of hybrid composite material shows porous surface. Circulation and position of lattices over the surface are like inside curved concaves with phase separation as shown in Figure 7.

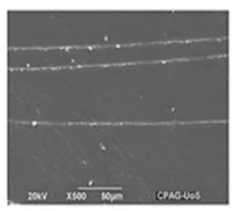

Organoclay

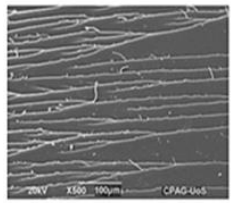

Organoclay

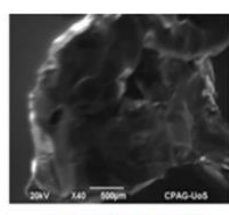

6 hours ultra-sonicated

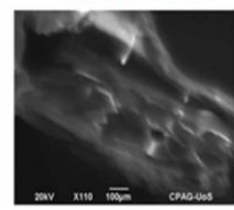

Fig. 6. SEM images of organoclay and 6-hour ultra-sonicated PS-PAA composite material

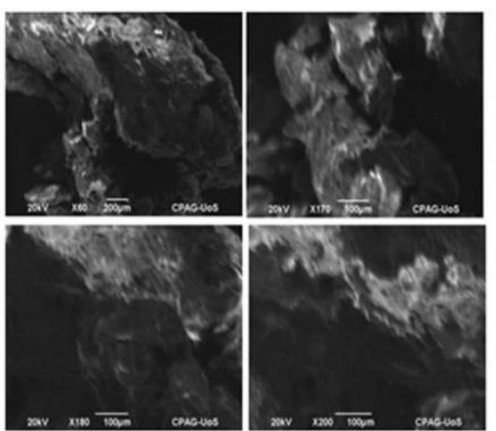

Fig. 7. SEM images of 12-hour ultra-sonicated PS-PAA hybrid composite material

\section{H. SEM Analysis Comparative}

Surface morphology declares the even distribution of organoclay (MMT) with PS-b-PAA composite material. SEM shows the phase separation and permeable surface of the hybrid composite material. MMT has diffusive properties at elevated temperatures. The material turns out to be significantly more grounded because of expanded support. To synthesize the composite material, filaments and layers of MMT are evenly adsorbed with help from the ultrasonic bath. Organic polymers with physically blended MMT have circulation, similarity of lattices with unjumbled spines and some-what rocky shape while, after 12 hours they show the inside curved concaves. With the assistance of SEM we decipher the morphological examinations at various optical zooms. The novel composite materials should be analyzed with SEM to elucidate the surface morphology, porosity, transport and network similitude which had been refined successfully. SEM pictures show permeable and penetrable surfaces (Figure8).

SEM pictures disentangle the morphological surface territories and the physical adsorption occurring through the shedding between the layers of block copolymer and MMT organoclay. (a)
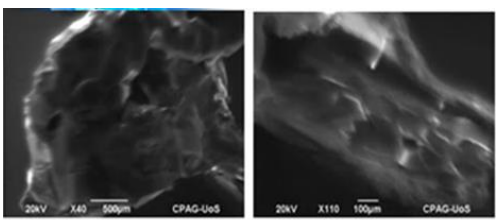

(b)

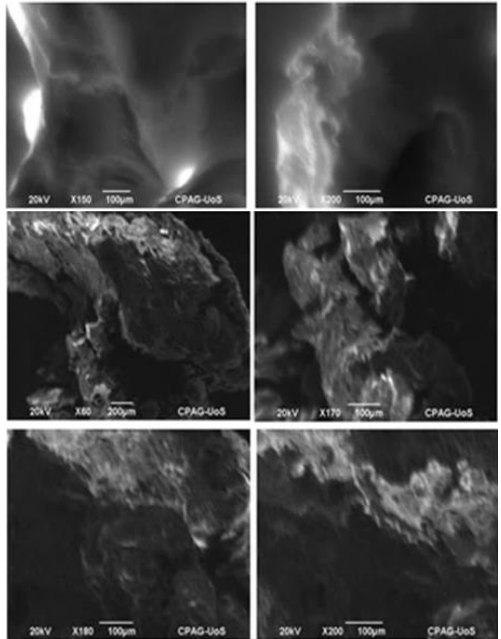

Fig. 8. Comparative 6-hour (a), and 12-hour (b) SEM analysis of hybrid composite

\section{CONCLUSIONS}

Polymeric composite materials of PS-b-PAA ultrasonicated with MMT had been combined through 6-hour and 12-hour ultrasonication at various interims. The composite material was subjected to UV-visible to confirm the finished structure. The antecedents and novel composite materials were studied by infrared and SEM techniques. The FT-IR spectrum confirms the incorporation of organoclay in the polymeric structure of PS and PAA with MMT. SEM pictures deciphered the surface morphology, porosity, circulation and similarity of framework and support in it, which had been accomplished effectively.

\section{REFERENCES}

[1] F. J. Doucet, C. Schneider, S. J. Bones, A. Kretchmer, I. Moss, P. Tekely, C. Exley, "The formation of hydroxyaluminosilicates of geochemical and biological significance", Geochimica et Cosmochimica Acta, Vol. 65, No. 15, pp. 2461-2467, 2001

[2] A. J. Erwin, W. Xu, H. He, K. Matyjaszewski, V. V. Tsukruk, "Linear and Star Poly (ionic liquid) Assemblies, Surface Monolayers and Multilayers", Langmuir,Vol. 33, No. 13, pp. 3187-3199, 2017

[3] C. Liu, H. Qin, P. T. Mather, "Review of progress in shape-memory polymers", Journal of Materials Chemistry, Vol. 17, No. 16, pp. 15431558,2007

[4] C. Sanchez, P. Belleville, M. Popall, L. Nicole, "Applications of advanced hybrid organic-inorganic nanomaterials from laboratory to market", Chemical Society Reviews, Vol. 40, No. 2, pp. 696-753, 2011

[5] H. Buhl, Advanced Aerospace Materials, Springer-Verlag, 2012

[6] Z. Spitalsky, D. Tasis, K. Papagelis, C. Galiotis, "Carbon nanotubepolymer composites: chemistry, processing, mechanical and electrical properties", Progress in Polymer Science, Vol. 35, No. 3, pp. 357-401, 2010

[7] R. N. Boyd, "Introduction", in: Stardust, Supernovae and the Molecules of Life, Springer Science \& Business Media, 2012

[8] C. E. Carraher Jr, Carraher's Polymer Chemistry, CRC Press, 2010

[9] Q. Chaudhry, L. Castle, R. Watkins, Nanotechnologies in Food, RSC Publishing, 2017 
[10] M. Zebarjadi, G. Joshi, G. Zhu, B. Yu, A. Minnich, Y. Lan, X. Wang, M. Dresselhaus, Z. Ren, G. Chen, "Power factor enhancement by modulation doping in bulk nanocomposites", Nano Letters, Vol. 11, No. 6, pp. 2225-2230, 2011

[11] D. Rawtani, Y. K. Agrawal, "Multifarious applications of halloysite nanotubes: A review", Reviews on Advanced Materials Science, Vol. 30, No. 3, pp. 282-295, 2012

[12] K. N. Prasad, B. Yang, M. Zhao, N. Ruenroengklin, Y. Jiang, "Application Of Ultrasonication Or High - Pressure Extraction Of Flavonoids From Litchi Fruit Pericarp", Journal of Food Process Engineering, Vol. 32, No. 6, pp. 828-843, 2009

[13] S. Q. Xie, J. Wan, B. R. Lu, Y. Sun, Y. Chen, X. P. Qu, R. Liu, “A nanoimprint lithography for fabricating SU-8 gratings for near-infrared to deep-UV application", Microelectronic Engineering, Vol. 85, No. 5, pp. 914-917, 2008

[14] J. Yang, S. Z. He, L. Q. Wang, "Dynamic balancing of a centrifuge: application to a dual-rotor system with very little speed difference", Modal Analysis, Vol. 10, No. 7, pp. 1029-1040, 2004

[15] J. Lamontagne, P. Dumas, V. Mouillet, J. Kister, "Comparison by Fourier transform infrared (FTIR) spectroscopy of different ageing techniques: application to road bitumens", Fuel, Vol. 80, No. 4, pp. 483488,2001

[16] J. Meng, "Understanding cultural influence on price perception: empirical insights from a SEM application", Journal of Product \& Brand Management, Vol. 20, No. 7, pp. 526-540, 2011 